\title{
Comparative Study on Travelling Salesman Problem
}

\author{
Yatharth Srivastav and J.K. Saini \\ Department of Mathematics, Chandigarh University-India \\ yatharthsrivastav01@gmail.com, drjatinder83@gmail.com
}

\begin{abstract}
Travelling Salesman Problem (TSP) is a kind of LPP to find minimum cost sequence in order to travel in each set of cities in a way that starting as well as ending should be on same city and each city is visited exactly one time. In this paper, we will compare different optimization algorithms working principle, and we will also discuss the advantages and limitations of all the optimization techniques.
\end{abstract}

Keywords:Travelling Salesman Problem (TSP), Ant Colony Optimization Algorithm (ACO), Genetic Algorithm (GA), Simulated Annealing Optimization (SA).

1.Introduction:TSP is a most wide ranged notorious problem in computational mathematics. This problem consists of a given set of cities and distance between them. TSP problem leads us to find the possible shortest route to visit all the given cities exactly once and returning to the origin. The origin of TSP is not particularly known. It is said that Euler gave the first ever instance of TSP in 1759 when he was trying to move a knight to each possible position on a chess board exactlyonce. The problem was formulated mathematically around 1800 by W.R. Hamilton, an Irish mathematician and a British mathematician Thomas Kirkman.

Currently there are different techniques and algorithm that are used to solve a TSP such as Ant colony algorithm, Genetic algorithm and Simulated Annealing optimization technique . In this paper we will examine these different techniques of optimization and compare for an overview.

\section{Ant Colony Optimization Algorithm (ACO):}

2.1Working Principle: The ACO algorithm is inspired by ants ${ }^{[1]}$. An ant usePheromone chemical to communicate with other ants in the colony to find an optimum path between colony and source of food located in the environment. This process is termed as Stigmergy. When ants locate the food, they use to spread randomly starting from colony to the source of food. In their path, they deposit Pheromone due to which they can trace the shortest distance easily between colony and food. Also, while returning, Pheromone is deposited in the path so that ants can return easily to the colony by the shortest path. After some time, this shortest path has more traffic, and all other longest paths are eliminated by ants.

The distance between two ants, pheromone deposition is the major key parameter in ACO algorithm.At first, ants starts their quest for food in a random manner. The ant chooses next node to visit by probabilistic equation ${ }^{[2]}$. Probability of ant ' $a$ ' going to node ' $j$ ' from node ' $I$ ' is followed by the eq. below;

$$
p_{i j}^{a}=\frac{\left(T_{j}\right)^{\alpha}\left(n_{i j}\right)^{\beta}}{\sum_{l \in N}\left(T_{j}\right)^{\alpha}\left(n_{i j}\right)^{\beta}} \quad \text { if } j \in N_{i}^{a}
$$


Where $N_{i}^{a}$ is closest node of ' $a$ ' which the ant has not visited yet. $\alpha$ is pheromone coefficient which controls the amount of pheromone contribution and is usually set as 0.1. Heuristic coefficient is $\beta$ that controls problem contribution and usually set between 2-5. The Number of ants is also usually set low. The shortest arc, which is used by the most ants, receives large amount of pheromone. Ants will use this arc to travel in future.

One important feature is pheromone update, that includes deposition of the chemical as well as its evaporation. In the time whenant moves from one to another node, its pheromone values are changed. The value of the first deposited pheromone on an arc is reduced by a factor which issaid to be pheromone evaporation that is constant. Pheromone deposit is when a small amount of pheromone is applied to each node that each ant is traversing. The equation for pheromone evaporation is as follows:

$$
T_{i j} \leftarrow(1-\rho) T_{i j}
$$

Where $\rho$ is symbolized as the rate of evaporation. Every ant drops pheromone on every node called as the pheromone deposit, follows the equation.

$$
T_{i j} \leftarrow T_{i j}+\sum_{a=1}^{m} \Delta T_{i j}^{a}
$$

Where $m$ denotes the total counting of ants. On a node, pheromone drop amount is given by $\Delta T_{i j}^{a}$ and calculated by $\frac{1}{C^{k}}$, where $C^{k}$ is the tour length travelled by $k^{t h}$ ant.

\subsection{Advantages of ant colony algorithm:}

1)Itcan be simply integrated with fellowalgorithms.

2)This technique is dependable.

3)When the graph changes dynamically, ant colony algorithms have an advantage over SA and GA approaches to similar problems, since they can continuously run and can be adjusted in real time change.

\subsection{Limitations of ant colony algorithm:}

1)This technique can solve certain problems with a great success rate, but their convergence cannot be proven.

2) The likelihood of dropping into optimal solution locality is very high as it adjusts pheromone in the direction that is more suitably available at a time.

\section{Genetic Algorithm:}

3.1Working Principle: A GA technique is based on natural selection that is one of the oldest and most efficient. It was first suggested by John Holland in the 1960s to research the natural evolution process and adaptation.The evolution theory ofCharles Darwininfluenced the GeneticAlgorithm ${ }^{[3]}$. One of the beat and suitable solution is chosen using the survival of the fittest method among the different solutions. To initiate the method, large amount of chromosomes are used and then each one of them is evaluated for fitness. Then the best and suitable chromosome is chosen to put through a crossover and mutation process to produce a new collection of chromosomes. To sustain population that evolves from generation to generation, GA employs three operations ${ }^{[4]}$. 
The $1^{\text {st }}$ one is the "Selection", based on "Survival of the Fittest" theorem. Quest begins with a population that is created at random and evolves over generations (iterations). The efficiency of the solutions is assessed using a fitness function.By using a selection method based on the fitness function, two solutions are chosen as parent solutions each time.

The $2^{\text {nd }}$ one is called"Crossover,"that is motivated by biological population mating. The operator transfers best surviving design characteristics from the parent to future population, resulting in a population with a higher average fitness value.

The $3^{\text {rd }}$ one is "Mutation," results in diversity of the population.This operation spontaneously triggers local changes to the new generation. Except for one or more modifications caused by the mutation process, the new generation is almost similar to the parent generation. These three operations are repeated continuously until population size of current generation equals the population size of previous generation. Then with new population, iteration process is initiated.

The new generation is far better than old generation because better solutions have a higher chance of being chosen for the process of crossover. The process is repeated until the consistency of improving the solution is very tough.

\subsection{Advantages of Genetic Algorithm:}

1)It often provides a solution, and the solution improves over time.

2)Multi-objective optimization is supported.

3)The technique is effective and more useful when analysis is mathematically available or we can say the problem has a poor and complex search space.

\subsection{Limitations of Genetic Algorithm:}

1)GA converge towards local optima if the fitness function is not properly defined.

2)It is difficult to operate on complex sets.

3)GA is unsuitable for constraint-based optimization problems.

\section{Simulated Annealing Algorithm:}

4.1Working Principle:The metallurgical method of annealing influenced Simulated Annealing. A substance is heated as well as cooled very slowly and turned into a solid state crystal, using the least amount of energy and the largest crystal size possible. This results in less defects. The atomic energies are raised by the heat and the atoms moves independently. As for cooling process, it allows the discovery of a new low-energy state. This annealing process necessitates precise temperature as well as cooling rate regulation.Every set of solutions reflects different system's internal energy. As machine cools, the sample approval conditions are narrowed to concentrate on enhancing movement.

As the temperature $(t)$ of the device rises, the probability $(p)$ of atrocious solutions decreases. Therefore, probability of approaching a good and easily feasible solution, which at a particular temperature will increase $(\Delta f)$ known as an objective function, can be calculated using a formula defined in Kirkpatrick's paper ${ }^{[5]}$.

$$
p(\Delta f, t)=\left\{\begin{array}{rr}
e^{\frac{-\Delta f}{t}} \Delta f \leq 0 \\
1, \quad \Delta f>0
\end{array}\right.
$$


The temperature at the starting and cooling rate for the SA algorithm must be set accordingly which temperature is reduced slower and chances to find an optimal solution is more. Indeed, Emile $\mathrm{H}$. Aarts demonstrated that this simulation should be done infinite times to find the best solution. Mostly, we can achieve a good cooling schedule by using $T_{0}$ as initial temperature, $\alpha$ as temperature decrement constant. Number of iterations should be fixed at each temperature.

\subsection{Advantages of Simulated Annealing Algorithm:}

1)It statistically ensures that the best solution is found.

2)Even for complex problems, coding is relatively simple.

3)SA can deal with results that are unordered and a variety of constraints.

5)It is adaptable because it is independent of the model's restrictive properties.

\subsection{Limitations of Simulated Annealing Algorithm:}

1) SA is not as useful when only few local minima are there.

2)SA is a meta-heuristic technique, which means it needs several options to be turned into an algorithm.

3)Accuracy of numbers used during implementation has a significant impact on the final product's quality.

\section{Conclusion:}

This paper compares the widely used TSP algorithms. We have compared three big approaches of TSP as definedin sections 2, 3 and 4 .

Ants use the ACO method in search of food sources. The technique called pheromone trail. GA is based on survival of the fittest principle and the law of biological reproduction, in which different operations are used to find the optimal solution. SA is based on the metallurgical annealing method, technique used to control the cooling of materials in order to minimize defect.

All three methods have a wide range of possible applications. All of these techniques gives us best results.

\section{References:}

1)M. Dorigo, L. Gambardella,

"Ant colonies for the Traveling salesman problem."

Biosystems 43, pp. 73-81, 19972.

2) M. Dorigo, T. Stutzle,

"Ant Colony optimization",

A Bradford book, MIT Press Cambridge, Massachusetts London, England, 2004.

3)N. Sureja, B. Chawda,

"Random Travelling Salesman problem using Genetic Algorithms." IFRSA's International Journal of Computing, Vol.2, issue 2, April 2012 
4)Melanie Mitchell., “

An Introduction to Genetic Algorithms"

MIT Press, Cambridge, MA, 1998

5)S. Kirkpatrick, C. D. Gelatt and M. P. Vecchi. “

Optimization by simulated annealing"

Science, Number 4598, vol. 220, 4598, pp.671-680, 13 May1983 